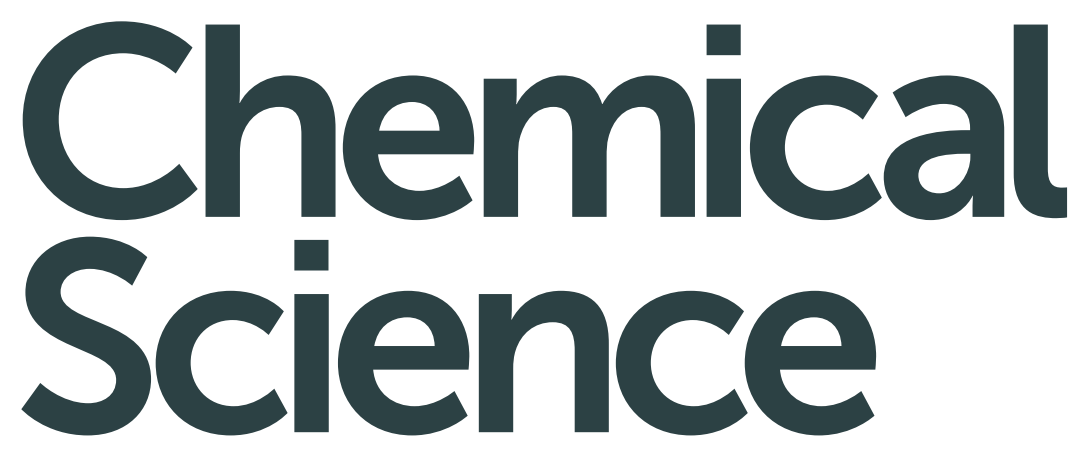

rsc.li/chemical-science

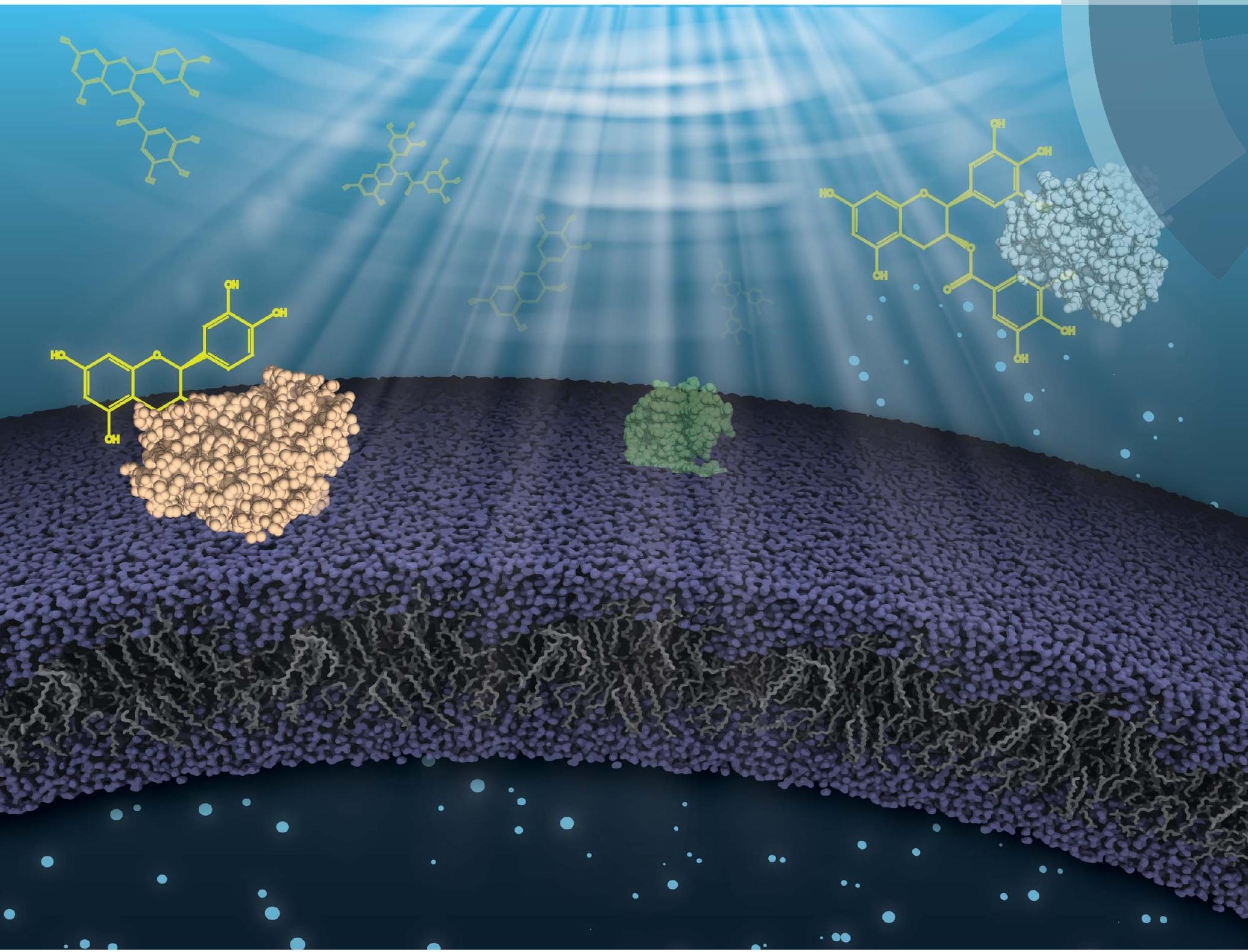

ISSN 2041-6539

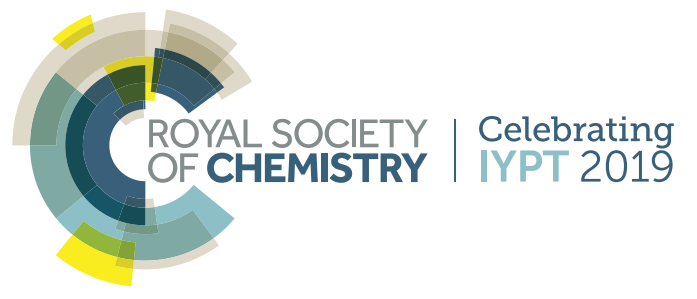

EDGE ARTICLE

Giuseppe Melacini et al.

Atomic resolution map of the soluble amyloid beta

assembly toxic surfaces

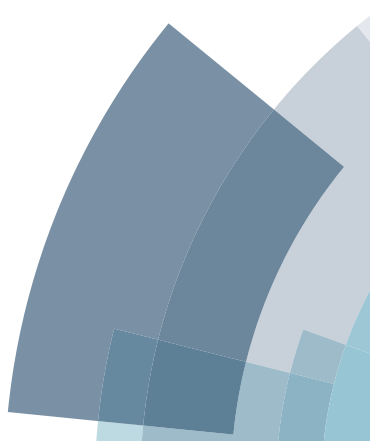




\section{Check for updates}

Cite this: Chem. Sci., 2019, 10, 6072

๑ All publication charges for this article have been paid for by the Royal Society of Chemistry

Received 19th March 2019

Accepted 19th May 2019

DOI: $10.1039 / \mathrm{c} 9 \mathrm{sc} 01331 \mathrm{~h}$

rsc.li/chemical-science

\title{
Atomic resolution map of the soluble amyloid beta assembly toxic surfaces $\uparrow$
}

\author{
Rashik Ahmed, (D) a Michael Akcan, ${ }^{a}$ Adree Khondker, ${ }^{b}$ Maikel C. Rheinstädter, (D) ${ }^{b}$ \\ José C. Bozelli, Jr, ${ }^{a}$ Richard M. Epand, (D) a Vincent Huynh, ${ }^{c}$ Ryan G. Wylie, (D) ${ }^{c}$ \\ Stephen Boulton, ${ }^{a}$ Jinfeng Huang, ${ }^{c}$ Chris P. Verschoor ${ }^{d}$ and Giuseppe Melacini (DD *ac
}

\begin{abstract}
Soluble amyloid beta assemblies $\left(A \beta_{n}\right)$ are neurotoxic and play a central role in the early phases of the pathogenesis cascade leading to Alzheimer's disease. However, the current knowledge about the molecular determinants of $A \beta_{n}$ toxicity is at best scant. Here, we comparatively analyze $A \beta_{n}$ prepared in the absence or presence of a catechin library that modulates cellular toxicity. By combining solution NMR with dynamic light scattering, fluorescence spectroscopy, electron microscopy, wide-angle X-ray diffraction and cell viability assays, we identify a cluster of unique molecular signatures that distinguish toxic vs. nontoxic $A \beta$ assemblies. These include the exposure of a hydrophobic surface spanning residues 17-28 and the concurrent shielding of the highly charged $\mathrm{N}$-terminus. We show that the combination of these two dichotomous structural transitions promotes the colocalization and insertion of $\beta$-sheet rich $A \beta_{n}$ into the membrane, compromising membrane integrity. These previously elusive toxic surfaces mapped here provide an unprecedented foundation to establish structure-toxicity relationships of $A \beta$ assemblies.
\end{abstract}

\section{Introduction}

While the etiology of Alzheimer's disease (AD) is complex and not fully understood, genetic mutations provide compelling evidence that the amyloid beta $(\mathrm{A} \beta)$ peptide plays a critical role in AD pathogenesis. ${ }^{1,2}$ Indeed, mutations in the genes encoding either the $\mathrm{A} \beta$ progenitor (i.e. the amyloid precursor protein or APP) or the APP processing enzyme (presenilin 1 and 2 genes) are sufficient to cause AD. ${ }^{1}$ Moreover, none of the familial $\mathrm{AD}$ mutations involve genes encoding for the tau protein. Instead, tau mutations enhance the deposition of neurofibrillary tangles i.e. the other neuropathological hallmark of $\mathrm{AD}$, but not amyloid plaques, and lead to different neuropathological disorders. ${ }^{3}$ These genetic signatures coupled with the observation that $\mathrm{A} \beta$ deposition precedes other biochemical and histopathological changes, including neurofibrillary tangle formation, ${ }^{4}$ provide evidence that tau aggregation occurs downstream to $A \beta$ aggregation. In addition, $A \beta$ clearance is

\footnotetext{
${ }^{a}$ Department of Biochemistry and Biomedical Sciences, McMaster University, Hamilton, ON, L8S 4M1, Canada.E-mail: melacin@mcmaster.ca

${ }^{b}$ Department of Physics and Astronomy, McMaster University, Hamilton, ON, L8S 4M1, Canada

${ }^{c}$ Department of Chemistry and Chemical Biology, McMaster University, Hamilton, ON, L8S 4M1, Canada

${ }^{d}$ Department of Health Research Methods, Evidence, and Impact (HEI), McMaster University, Hamilton, ON, L8S 4M1, Canada

$\dagger$ Electronic supplementary information (ESI) available: Methods, ${ }^{15} \mathrm{~N}-\mathrm{DEST}$ profiles, and additional statistical analyses. See DOI: 10.1039/c9sc01331h
}

controlled by one of the most significant risk factors for late onset $\mathrm{AD}$, i.e. APOE4..$^{5}$ Given the genetic link between $\mathrm{A} \beta$ and $\mathrm{AD}$, one of the main hypotheses proposed to explain $\mathrm{AD}$ pathogenesis is the amyloid cascade. The amyloid hypothesis posits that neuronal death in $\mathrm{AD}$ patients is associated with the increased production, self-association and accumulation of $\mathrm{A} \beta$ in the brain. ${ }^{2}$

Since it was originally postulated, the generality of the amyloid cascade hypothesis has been challenged because $A \beta$ plaque burden correlates poorly with cognitive dysfunction. ${ }^{6}$ However, this inconsistency has been reconciled by considering that soluble oligomers and protofibrils formed during the selfassociation cascade towards mature fibrils are neurotoxic $\mathrm{c}^{7}$ and better correlate with cognitive impairment in the early stages of $\mathrm{AD}^{8}{ }^{8}$ Moreover, the neurotoxicity of $\mathrm{A} \beta$ oligomers has been linked to tau hyperphosphorylation, ${ }^{9}$ providing further evidence in support of the upstream role of soluble $A \beta$ assemblies in the $\mathrm{AD}$ pathogenesis cascade. ${ }^{1}$

The central role of soluble $A \beta$ oligomers and protofibrils in $\mathrm{AD}$ has prompted substantial efforts to identify the molecular determinants of neurotoxicity in soluble $A \beta$ assemblies $\left(A \beta_{n}\right.$, where $n$ represents the number of $\mathrm{A} \beta$ molecules comprising the assembly). ${ }^{10-22}$ Unfortunately, given the transient and heterogeneous nature of $A \beta$ intermediates, characterization of their structure and properties has been challenging. Despite these hurdles, it has been possible to delay the growth of aggregation intermediates to an extent sufficient to enable structural elucidation. For example, Ahmed et al. have shown that toxic $\mathrm{A} \beta_{42}$ 
oligomers stabilized through low temperature and salt conditions are largely disordered, but exhibit a turn conformation reminiscent of protofibrils and fibrils. ${ }^{20}$ In contrast, for the other major isoform of $\mathrm{A} \beta$, i.e. $\mathrm{A} \beta_{40}$, toxic oligomers adopt parallel, in-register $\beta$-sheets. ${ }^{21}$ While these studies have provided an initial framework to define structural features of toxic $\mathrm{A} \beta_{n}$, the location of the "toxic $\mathrm{A} \beta_{n}$ surfaces" remains unclear. Mapping such surface sites is critical as the exposure of toxic surfaces shared by multiple soluble $A \beta_{n}$ species has been hypothesized to be one of the main causes of $A \beta_{n}$ toxicity. ${ }^{1,23}$

Exposure of these toxic surfaces is thought to facilitate interactions with multiple cellular components, including membranes, which underlie key pathogenic steps in the progression of $\mathrm{AD} .^{\mathbf{1 , 2 2 , 2 4 - 2 6}}$ In fact, extracellular $\mathrm{A} \beta$ oligomers are known to perturb biological and biomimetic membranes at multiple levels. The oligomers can (i) bind to membranes causing local perturbations, ${ }^{19,27}$ (ii) form annular structures that insert into the membrane and affect ion homeostasis ${ }^{\mathbf{1 6 , 1 8 , 1 9}}$ and (iii) bind to membrane receptors altering signal transduction pathways. ${ }^{28}$ Similar hypotheses have been proposed to explain the neurotoxicity of $A \beta$ protofibrils, ${ }^{17}$ although the latter have been shown to act also through detergent-like permeabilization and eventual fragmentation of the membrane. ${ }^{19}$ While these results highlight critical aspects of $A \beta$-membrane interactions, the "toxic surfaces" that enable key interactions with the membrane, as well as the underlying mechanism, remain elusive.

As a further step towards dissecting the molecular determinants of soluble $\mathrm{A} \beta_{n}$ toxicity and mapping the toxic $\mathrm{A} \beta_{n}$ surfaces, here we systematically investigate a library of $A \beta_{40}$ assemblies sampling different degrees of cellular toxicity. To this end, we first stabilized canonical, toxic $A \beta_{40}$ assemblies through desalting and low temperature ${ }^{29}$ and then treated them with a diverse set of catechins, ranging from (-)-epigallocatechin-3-gallate (EGCG), which remodels A $\beta$ into nontoxic structures, ${ }^{30}$ to (-)-epicatechin (EC), which is expected to detoxify $A \beta$ only partially. We then profiled our soluble $A \beta$ library through multiple complementary techniques with different degrees of spatial resolution, including extrinsic fluorescence, electron microscopy, dynamic light scattering, wide-angle X-ray diffraction and NMR spectroscopy. Unlike previous attempts to dissect the toxicity determinants of $A \beta$ assemblies, ${ }^{20,21}$ here we characterize representative soluble $A \beta$ assemblies from our library both in the absence and presence of model membranes.

The comparative analysis of our soluble $A \beta_{40}$ library reveals a cluster of key toxicity determinants and the associated mechanism of action. We discovered that toxicity scales proportionally to the enhanced hydrophobic exposure of $A \beta_{40}$ assemblies and their ability to interact with $\mathrm{A} \beta$ monomers and cell membranes. The hydrophobic region spanning residues 17-28 is more accessible to monomer recognition in toxic $\mathrm{A} \beta_{n}$ relative to $\mathrm{A} \beta_{n}$ with reduced cellular toxicity. Moreover, whereas increased exposure of hydrophobic residues is required for toxicity, we find that shielding of the highly charged $\mathrm{N}$ terminus, i.e. residues $<12$, from $\mathrm{A} \beta$ monomer recognition enhances the toxicity of $A \beta_{n}$. These toxic $A \beta_{n}$ surfaces are critical for the binding of $A \beta_{n}$ to lipid membranes and for forming membrane-embedded $\beta$-sheet structures, which compromise the integrity of the cell membrane. The resulting model provides a foundation to start defining structure-toxicity relationships of $\mathrm{A} \beta$ assemblies.

\section{Results and discussion}

\section{An $\mathbf{A} \beta_{40}$ assembly library that samples a cytotoxicity gradient}

As a first step towards dissecting the determinants of $\mathrm{A} \beta_{40}$ toxicity, we prepared a library of soluble $\mathrm{A} \beta_{n}$ spanning a cytotoxicity gradient. For this purpose, we incubated canonical (non-treated) $\mathrm{A} \beta_{n}$ with a collection of seven distinct catechins expected to remodel to varying extents the pre-existing soluble toxic $\mathrm{A} \beta_{n}$ into less toxic species ${ }^{30-32}$ (ESI Fig. S1, $\dagger$ Methods). Out of this $\mathrm{A} \beta_{n}$ library, we selected a sub-set of representative $\mathrm{A} \beta$ assemblies (i.e. those formed in the presence of the EC, (-)-epigallocatechin (EGC) and EGCG catechins) for toxicity profiling in a human retinal pigment epithelial (RPE1) cell line. The state of the RPE1 cells was first monitored by performing PrestoBlue assays, which rely on the reductive potential of the cell as a proxy of cellular viability. ${ }^{33}$ Relative to mock (i.e. PBS delivery vehicle), canonical $\mathrm{A} \beta_{n}$ significantly decrease cellular viability (Fig. 1a, black vs. grey). In contrast, $\mathrm{A} \beta_{n}$ formed in the presence of catechins are less effective in reducing cellular viability, in the order EC (Fig. 1a, green), EGC (Fig. 1a, yellow) and EGCG (Fig. 1a, maroon), for which no significant difference is detected compared to mock (Table S1†). Only negligible changes in cellular viability were observed for cells treated with catechins alone (Fig. 1a, dark green, orange and brown).

We also stained RPE1 cells with the necrotic cell marker propidium iodide (PI), which binds to DNA in cells with severely compromised membranes. ${ }^{34}$ The RPE1 cells were also counterstained with the nuclear marker Hoechst $333422^{35}$ to show that non-specific PI-staining is negligible under our conditions, as indicated by the purple $v s$. red fluorescence for PI in merged $v s$. separate panels, respectively (Fig. 1b). Fluorescence microscopy images of RPE1 cells treated with canonical $A \beta_{n}$ indicate prominent staining with PI (Fig. 1b). In contrast, $A \beta_{n}$ formed in the presence of catechins exhibit remarkably less PI staining (Fig. 1b), following the same EC < EGC < EGCG ranking as the cellular viability assay (Fig. 1a). Overall, these results suggest that the $A \beta$ assemblies in our library elicit different levels of cellular dysfunction and cell death. Hence, the comparative analysis of such $A \beta$ aggregates is anticipated to reveal key molecular determinants of soluble $A \beta$ toxicity.

\section{The $A \beta$ assembly library spans a wide distribution of sizes, hydrophobic solvent exposures and cross $\beta$-sheet contents}

We first evaluated how our catechin library remodels the distribution of $\mathrm{A} \beta$ assemblies. For this purpose, the relative populations of the NMR visible low MW A $\beta$ species (e.g. monomers) were gauged through residual ${ }^{1} \mathrm{H}$ NMR intensities (Fig. 1c), while the NMR invisible $\mathrm{A} \beta_{n}$ were probed by dynamic light scattering (DLS) (Fig. 1d and e). While it is important to complement these data with size estimations through other 
a
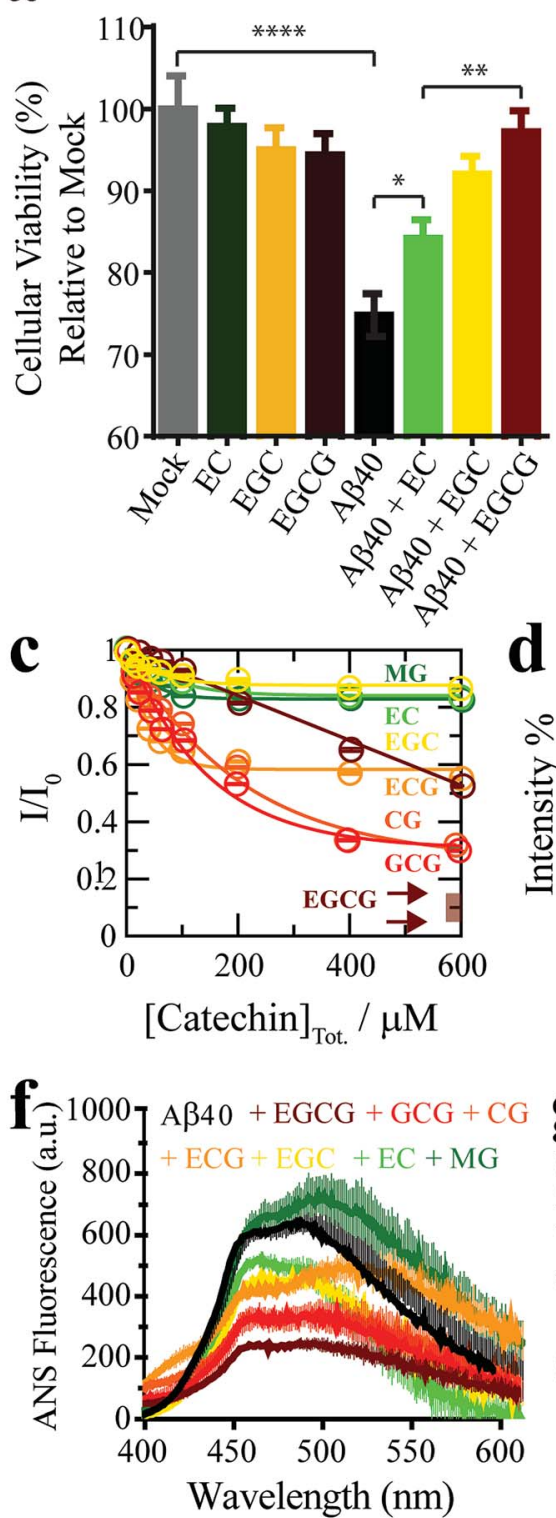

b
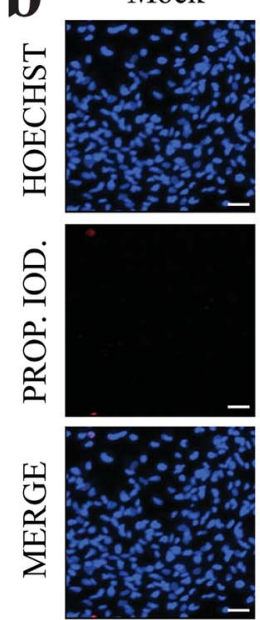

d

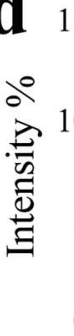

15
10
5

0
0
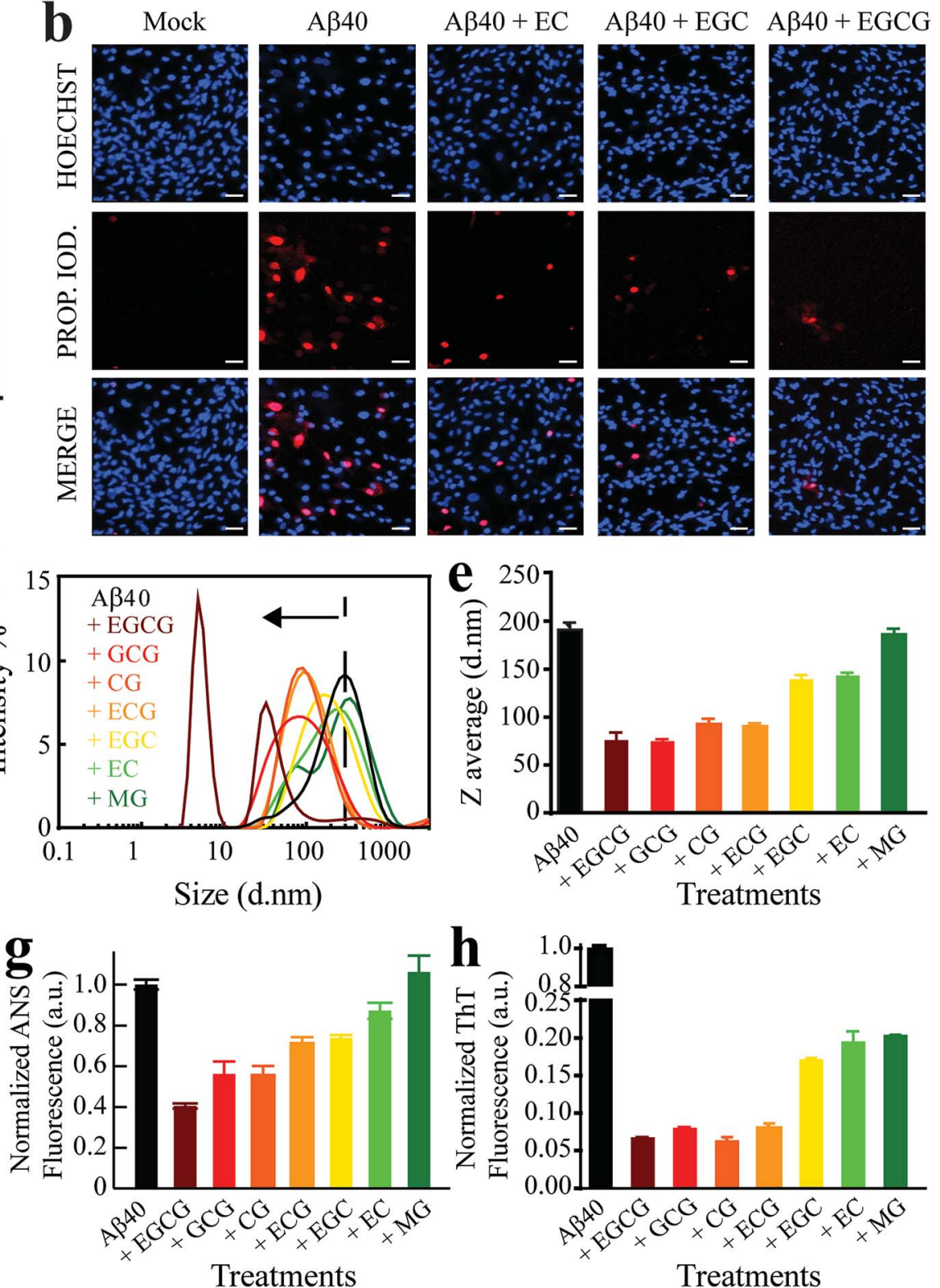

Fig. 1 The $A \beta_{n}$ library samples a wide-distribution of toxicity, hydrophobic exposure, cross $\beta$-sheet content and sizes. (a) Mitochondrial activity of retinal pigment epithelial (RPE1) cells after treatment with representative $A \beta_{40}$ assemblies and associated controls, as monitored by the reduction of resazurin using the PrestoBlue assay. ${ }^{33}$ The data reported show the mean and standard deviation of technical replicates. One-way ANOVA and subsequent Tukey's post-hoc test was used to determine statistical significance between treatments and mock (1X PBS delivery solution), with *, $* *$ and **** representing $p$-values of $0.05,0.01$ and $<0.0001$, respectively. (b) Representative fluorescence microscopy images of RPE1 cells (scale bar, $50 \mu \mathrm{m}$ ), showing intracellular Hoechst 33342 and propidium iodide fluorescence after incubation with selected $A \beta_{40}$ assemblies. (c) Normalized $A \beta_{40}$ methyl intensity losses upon catechin addition relative to the state in the absence of catechins. (d) Size distribution of $A \beta_{40}$ assemblies in the absence (black) and presence of catechins (coloured as per legend) as determined by intensity measurements in dynamic light scattering experiments. (e) Z-average of the $A \beta_{40}$ assemblies in (d). (f) ANS fluorescence spectra of $A \beta_{40}$ assemblies in the absence (black) and presence of catechins (colour coded as per the legend). (g) ANS fluorescence intensities at $454 \mathrm{~nm}$ for the samples in (k), normalized to the intensity for $A \beta_{40}$ alone. (h) Thioflavin T fluorescence intensities at $485 \mathrm{~nm}$ of $A \beta_{40}$ assemblies in the absence (black) and presence of catechins (coloured as per legend) normalized to the intensity of canonical assemblies.

means, such as TEM (vide infra), interestingly, we observed that all catechins in our library reduce the populations of both the $\mathrm{A} \beta$ monomers (Fig. 1c) and the $\mathrm{A} \beta$ assemblies at the opposite end of the molecular weight (MW) distribution (Fig. 1d and e). These results suggest that the $A \beta$ species at the extremes of the probability distribution are converted by the catechins into $A \beta$ species with intermediate MW. However, the extent of this remodeling is markedly catechin-dependent with (-)-catechin3-gallate (CG) leading to large reductions in both the monomer and high MW populations (Fig. 1c-e) and methyl-3,4,5trihydroxybenzoate (MG) causing only marginal changes (Fig. 1c-e). 
We also investigated the surface hydrophobicity of the $A \beta$ assemblies formed under our conditions, as exposed hydrophobic surfaces have been associated with toxicity for another amyloidogenic system. ${ }^{36}$ The surface hydrophobicity of $A \beta_{n}$ was probed through 8-anilino-1-naphthalenesulfonic acid (ANS) fluorescence, which exhibits a characteristic blueshift and enhancement in fluorescence intensity upon binding exposed hydrophobic sites. A substantial enhancement in ANS fluorescence was observed for canonical $\mathrm{A} \beta_{n}$ (Fig. 1f and g, black), whereas the extent of such enhancement is significantly reduced for most catechin-treated $A \beta_{n}$ (Fig. $1 \mathrm{f}$ and $\mathrm{g}$, coloured). Notably, the measurements of the catechin-treated $A \beta_{n}$ surface hydrophobicity (Fig. 1f and g) rank in the same order as the cell toxicities (Fig. 1a), suggesting that exposed hydrophobic surfaces are a key determinant of $A \beta_{n}$ toxicity.

Another unique signature of amyloids is the formation of extensive cross $\beta$-sheets, as reported by the fluorescent dye Thioflavin $\mathrm{T}$ (ThT). Canonical, toxic $\mathrm{A} \beta_{n}$ exhibit significant ThT fluorescence in comparison to catechin-remodeled $\mathrm{A} \beta_{n}$ (Fig. 1h). While the decreased ThT fluorescence in the presence of EGCG is in agreement with previous observations, ${ }^{30,37,38}$ our data on the extended catechin library reveal that other catechins also preserve the ability to destabilize intermolecular $\beta$-sheets and/or outcompete ThT. Hence, ThT-responsive $\beta$-amyloids do not appear to correlate with cytotoxicity as well as the observables reported above i.e. size and hydrophobic exposure. Indeed, solvent accessible hydrophobic moieties are one of the main drivers for $\mathrm{A} \beta$-membrane interactions, which in turn have been proposed as a key determinant of the cytotoxicity associated with $\mathrm{A} \beta .^{39}$ This hypothesis is supported by our propidium iodide results, which indicate that toxic $A \beta_{n}$ severely compromise the integrity of cell membranes (Fig. 1b). To further corroborate this hypothesis, we evaluated the interactions between a representative subset of our $A \beta_{n}$ library and biomimetic membranes (small unilamellar vesicles, SUVs).

\section{Toxic $A \beta$ assemblies co-localize, bind and insert into biomimetics membranes}

We profiled the membrane interactions of selected $A \beta$ assemblies from our library that report on representative regions of our toxicity scale, i.e. the canonical as well as the EC- and EGCGremodeled $A \beta_{n}$ (Fig. 1a). For this purpose, SUVs composed of a mixture of DOPE : DOPS : DOPC lipids were prepared with an effective size distribution ranging from $\sim 10-100 \mathrm{~nm}$ and an average diameter of $\sim 34 \mathrm{~nm}$ (Fig. $2 \mathrm{a}$ and $\mathrm{b}$ ). Prior to the addition of the $A \beta_{n}$ library to the SUVs, we characterized the morphology of the $A \beta_{n}$ by TEM to ensure that significant catechin-induced remodeling occurs. Indeed, compared to canonical $\mathrm{A} \beta_{n}$, which primarily adopt "worm-like" protofibrils (Fig. 2c, top left panel), we observed both spherical assemblies and amorphous aggregates in the presence of EGCG (Fig. 2c, top right panel). The latter of the two species has been reported to be an intermediate in the formation of the former. ${ }^{23}$ In contrast, the EC-remodeled $A \beta_{n}$ displays features of both canonical and EGCG-remodeled $A \beta_{n}$, albeit more closely resembling the canonical $\mathrm{A} \beta_{n}$ (Fig. $2 \mathrm{c}$, top center panel). Having confirmed that catechin-induced remodeling of $A \beta_{n}$ occurs, we then evaluated to what extent the $A \beta_{n}$ library interacts with SUVs.

TEM images reveal that canonical A $\beta_{n}$ significantly colocalize with SUVs. For example, it is possible to observe select $\mathrm{A} \beta_{n}$ copositioned with the lipids (Fig. 2c, bottom left panel). Similar to the canonical $\mathrm{A} \beta_{n}$, EC-remodeled $\mathrm{A} \beta_{n}$ are also somewhat colocalized with the SUVs (Fig. 2c, bottom center panel). However, in stark contrast to both the canonical and ECremodeled $\mathrm{A} \beta_{n}$, the EGCG-remodeled $\mathrm{A} \beta_{n}$ are on average spatially distinct from the SUVs (Fig. 2c, bottom right panel).

To complement the TEM data on canonical vs. catechinremodeled $\mathrm{A} \beta_{n}$-membrane interactions, we performed ${ }^{15} \mathrm{~N}$ transverse relaxation $\left(\mathrm{R}_{2}\right),{ }^{1} \mathrm{H}$-based saturation transfer difference (STD) as well as ${ }^{15} \mathrm{~N}$-Dark State Exchange Saturation Transfer (DEST) NMR experiments, which collectively probe the interactions of $\mathrm{A} \beta$ with high MW (HMW) species, including SUVs, $A \beta_{n}$ and their complexes, through the lens of the NMR visible $A \beta$ monomers (Fig. $3 \mathrm{a}-\mathrm{g}$ ). ${ }^{29,40-48}$ Upon addition of SUVs to the canonical $\mathrm{A} \beta_{n}$, we observed marked enhancements in $\mathrm{R}_{2}$ and STD (Fig. 3a and b), consistent with the $A \beta_{n}$-membrane interactions revealed by TEM (Fig. 2c). The SUV-induced changes in $\mathrm{R}_{2}$ and ${ }^{1} \mathrm{H}$-based saturation transfer are more pronounced for the residues in the $\beta 1$ (residues $12-24$ ) and $\beta 2$ regions (residues $30-40$ ) than for the N-terminal moiety (residues $<12$ ), indicating that the $\beta 1$ and $\beta 2$ segments serve as key hot-spots of the SUV-A $\beta$ interactions under our experimental conditions. This conclusion is independently confirmed by the comparative analysis of the ${ }^{15} \mathrm{~N}$-DEST data (Fig. $3 \mathrm{~g}-\mathrm{m}$ ).

Residues in direct contact with the $\mathrm{A} \beta_{n} / \mathrm{SUV}$ surface typically display an attenuation of the residual monomer DEST signal, leading to broadening of the residue-specific ${ }^{15} \mathrm{~N}$-DEST $v$ s. offset profile relative to amino acids for which the monomer is disengaged from the $A \beta_{n} /$ SUV surface. ${ }^{31}$ Such broadening of the ${ }^{15} \mathrm{~N}$-DEST profile is quantitatively measured through the $\Theta$ parameter at intermediate ${ }^{15} \mathrm{~N}$-continuous wave (CW) offsets, ${ }^{40,49,50}$ as explained in the Methods. Consistent with the $\mathrm{R}_{2}$ and STD data (Fig. 3a and b), upon SUV addition to canonical $\mathrm{A} \beta_{n}$, major DEST $v s$. offset profile broadening and corresponding $\Theta$ enhancements are observed for the $\beta 1$ and $\beta 2$ regions (Fig. 3c-g and k; ESI Fig. S3†). A similar observation applies to the addition of SUVs to EC-remodeled $\mathrm{A} \beta$, which on average display a pattern comparable to canonical $\mathrm{A} \beta_{n}$ (Fig. $3 \mathrm{~h}$ and $\mathrm{l} v$ s. Fig. $3 g$ and k; ESI Fig. S4†). Conversely, the EGCG-remodeled A $\beta$ do not exhibit significant $\beta 1$ and $\beta 2$ enhancements as compared to canonical and EC-remodeled A $\beta$ (Fig. $3 \mathrm{i}$ and m; ESI Fig. S5 $\dagger$ ), in excellent agreement with the TEM observations. While the combination of our TEM and ${ }^{15} \mathrm{~N}$-based NMR experiments reveal key differences in $A \beta$-membrane interactions between the less toxic EGCG-remodeled $\mathrm{A} \beta$ and the more toxic canonical and EC-remodeled $A \beta$, they do not provide direct insight about whether $\mathrm{A} \beta_{n}$ inserts into the membrane and about the structural features of membrane-embedded $A \beta_{n}$. To this end, we conducted wide-angle X-ray diffraction (WAXD) experiments in the presence of model membranes for $A \beta$ assemblies at representative regions of our toxicity scale (Fig. 2d-1).

The WAXD two-dimensional intensity maps (Fig. 2e) were modeled with a series of Lorentzian fits (Methods) to derive 
A DOPE:DOPS:DOPC SUVS C

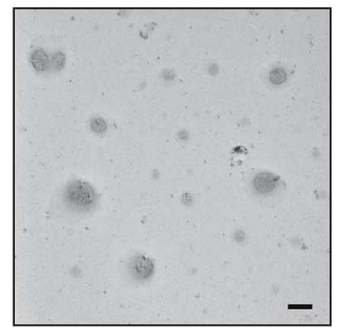

b

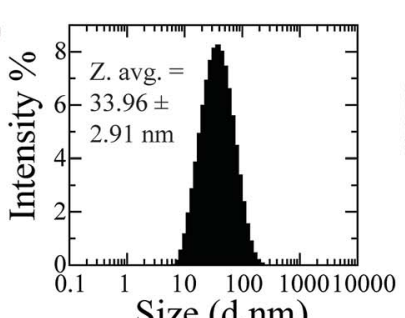

d Size (d.nm)
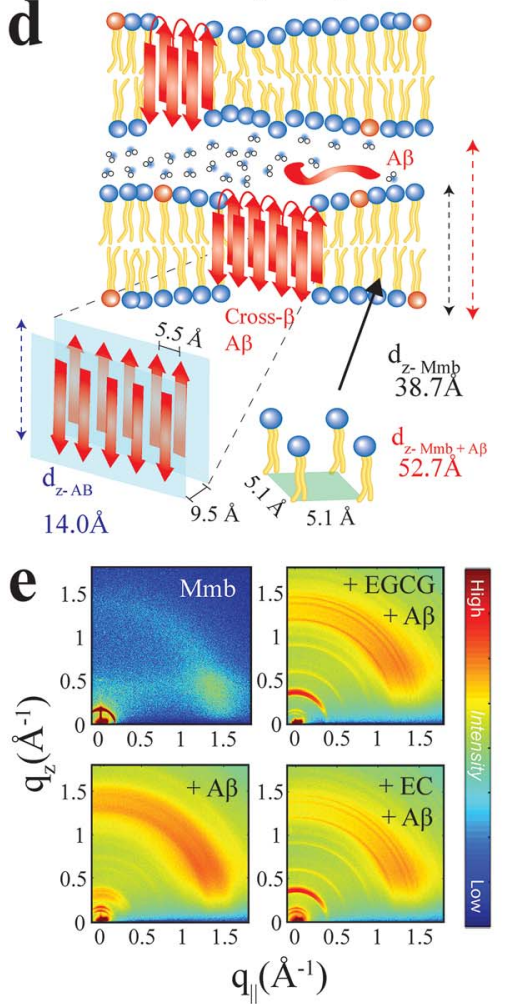

C $\quad \mathrm{A} \beta 40$
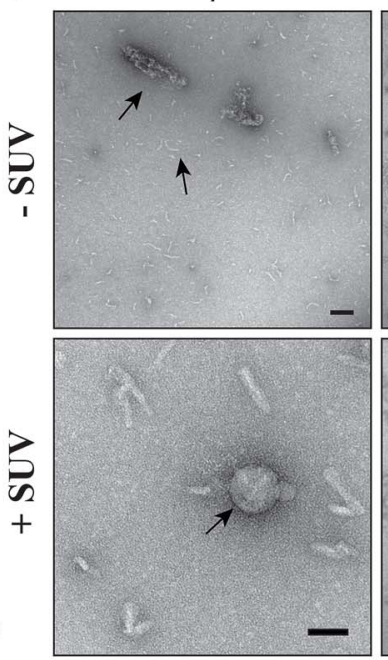

f

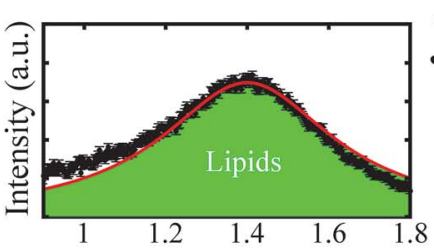

g

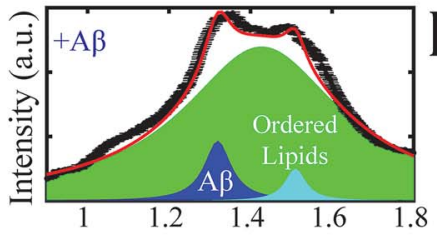

h

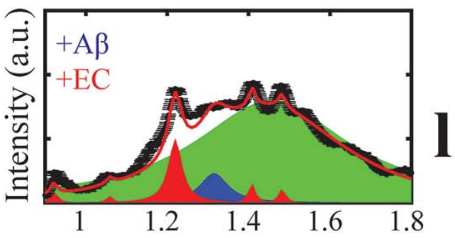

i

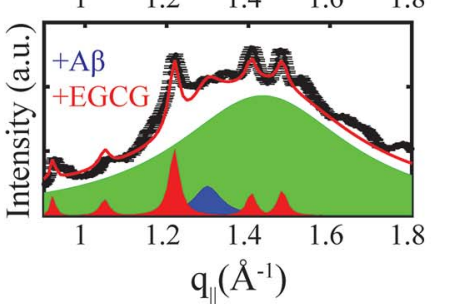

$+\mathbf{E C}$
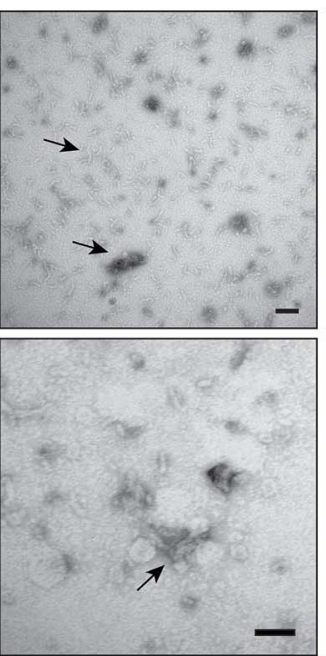
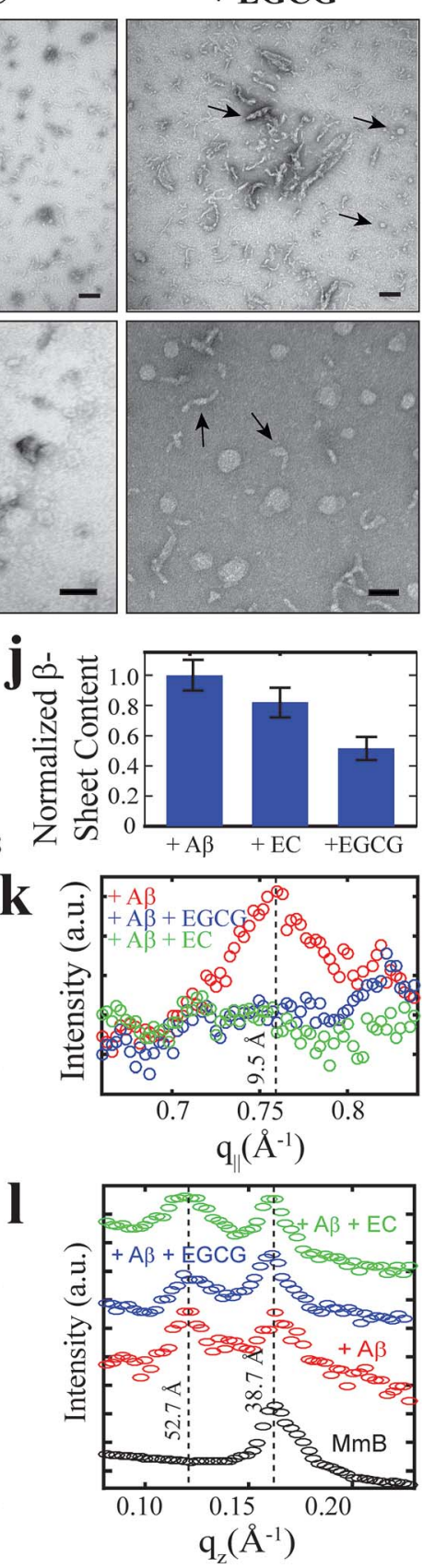

Fig. 2 Localization and insertion of $A \beta_{40}$ assemblies into model membranes. (a) Negative-stain TEM image of $800 \mu M$ DOPE : DOPS : DOPC SUVs. (b) Size distribution of SUVs shown in (a) as determined through dynamic light scattering intensity measurements. (c) Negative-stain TEM images of $A \beta_{40}$ assemblies in the absence and presence of EC and EGCG and the same assemblies treated with the SUVs in (a) and (b). All scale bars correspond to $100 \mathrm{~nm}$. (d) Schematic summary of the information extracted from wide-angle X-ray diffraction experiments. (e) Complete two-dimensional intensity maps of the X-ray diffraction data with both in-plane and out-of-plane features. (f-i) In-plane (q $\|$ ) diffraction patterns (black line) and fitted Lorentzian peaks (coloured peaks) for DOPE : DOPS : DOPC lipids (green peaks) in the absence and presence of A $\beta_{40}$ assemblies (blue peaks) with and without catechins (red peaks). Red lines indicate total fits derived from the summation of component peaks. (j) Normalized population of membrane-embedded $\beta$-sheet assemblies relative to canonical $A \beta_{40}$ assemblies, derived through the integration of blue $A \beta$ peaks in $(f-i)$. $(k)$ In-plane $\left(q_{\|}\right)$diffraction patterns highlighting the cross- $\beta$ inter-sheet signal intensity, which correspond to the $9.5 \AA$ spacing between $\beta$-sheets shown in (d). (l) Out-of-plane $\left(q_{z}\right)$ diffraction patterns depicting the membrane lamellar spacing (panel d, dashed black and red lines corresponding to 38.7 and $52.7 \AA$, respectively) in the absence (black) and presence (coloured as per legend) of $A \beta_{40}$ assemblies.

structural features both in-plane ( $\mathrm{q}_{\|}$, Fig. 2f-k) and out-of-plane $\left(\mathrm{q}_{\mathrm{z}}\right.$, Fig. 2l) of the membrane. For the lipid sample in the absence of $\mathrm{A} \beta_{n}$, in-plane and out-of-plane Bragg peaks were observed at $1.41 \AA^{-1}$ (Fig. 2f) and $0.17 \AA^{-1}$ (Fig. 2l, black), respectively, corresponding to the formation of bilayer stacks with an effective bilayer width of $38.7 \AA$ and a $5.1 \AA$ spacing 

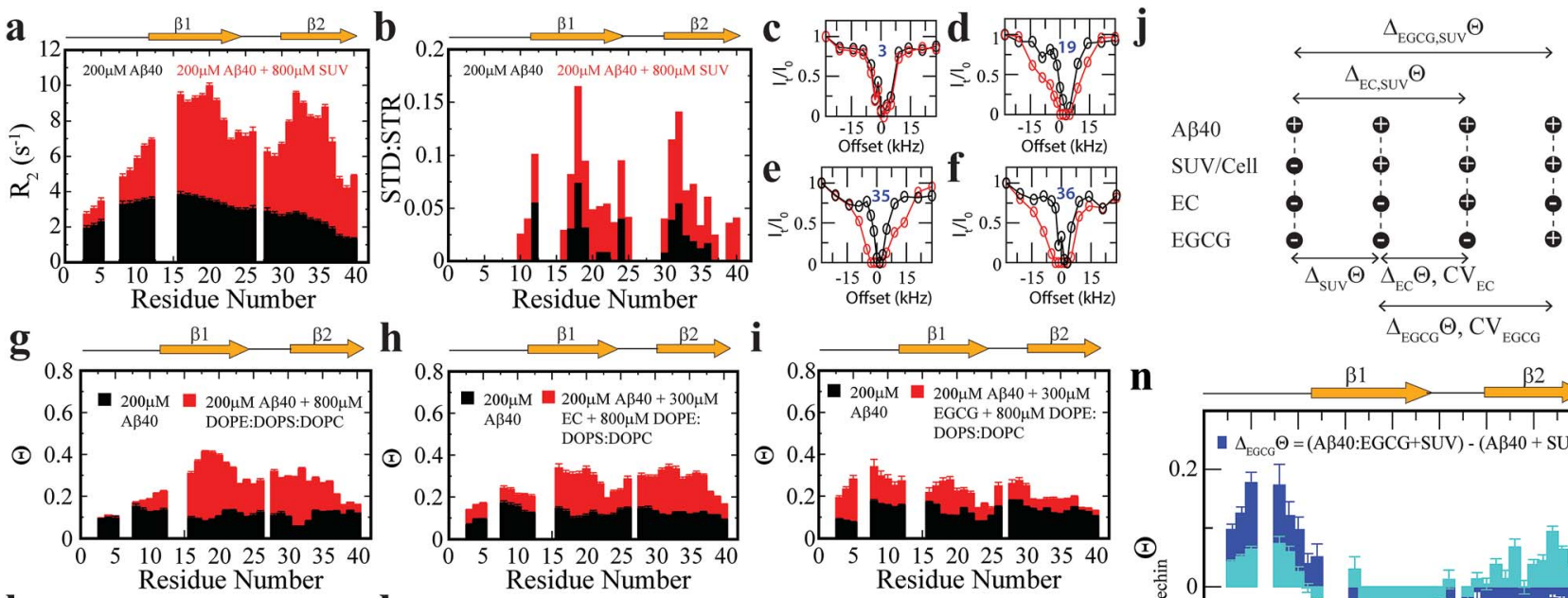

$\mathbf{n}$

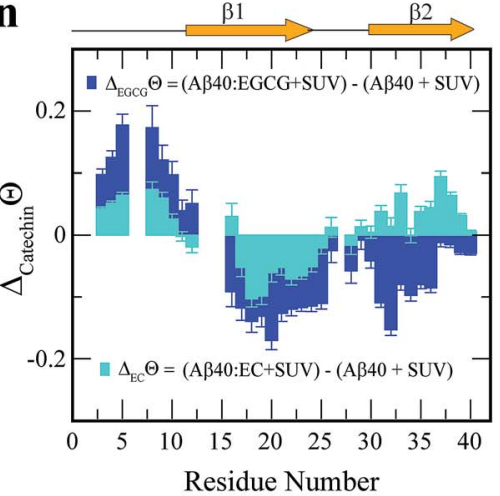

Fig. 3 Exchange dynamics of $A \beta_{40}$ monomers on the surface of soluble $A \beta_{40}$ assemblies and model membranes. (a) ${ }^{15} \mathrm{~N}-\mathrm{R}_{2}$ and (b) MeSTDHSQC for the canonical $A \beta_{40}$ assemblies in the absence (black) and presence (red) of DOPE : DOPS : DOPC SUVs. (c-f) Representative ${ }^{15} \mathrm{~N}-\mathrm{DEST}$ profiles for the samples shown in (a). (g) ${ }^{15} \mathrm{~N}-\Theta$ profiles for the samples shown in (a), colour coding is as per legend. (h) ${ }^{15} \mathrm{~N}-\Theta$ profiles for canonical $A \beta_{40}$ assemblies in the absence (black) and presence (red) of EC followed by DOPE : DOPS : DOPC SUV addition. (i) ${ }^{15} \mathrm{~N}-\Theta$ profiles for canonical $A \beta_{40}$ assemblies in the absence (black) and presence (red) of EGCG followed by DOPE : DOPS : DOPC SUV addition. (j) Definition of key differentials in the ${ }^{15} \mathrm{~N}$-DEST measurements and the corresponding normalized cellular viabilities. (k) Difference between the $\Theta$ profiles shown in (g). The dashed red line indicates the average $\Theta$ value. (l) Difference in the $\Theta$ profiles shown in $(\mathrm{h})$. ( $\mathrm{m}$ ) Difference between the $\Theta$ profiles shown in (i). (n) ${ }^{15} \mathrm{~N}-\Theta$ difference profiles for (h, red) vs. (g, red) (cyan) and (i, red) vs. (g, red) (blue).

between individual lipids (Fig. 2d). Addition of canonical $\mathrm{A} \beta_{n}$ to these lipid bilayers results in additional in-plane features at 1.32 $\AA^{-1}$ (Fig. $2 \mathrm{~g}$, blue) and $0.76 \AA^{-1}$ (Fig. $2 \mathrm{k}$, red), indicating the presence of membrane-embedded $A \beta_{n}$ adopting laminated $\beta$ sheets with $5.5 \AA$ A spacing between adjacent $\beta$-strands and $9.5 \AA$ between $\beta$-sheet layers (Fig. 2d). Interestingly, we observe an additional peak at $1.51 \AA^{-1}$ (Fig. $2 \mathrm{~g}$, cyan) corresponding to highly ordered lipids likely in the regions interfacing with the embedded $\mathrm{A} \beta_{n}$. Moreover, an out-of-plane diffraction pattern is observed at $\sim 0.12 \AA^{-1}$ (Fig. 2l, red) consistent with the presence of $A \beta$ not embedded into the bilayer (Fig. $2 \mathrm{~d}$ ).

Compared to canonical $\mathrm{A} \beta_{n}$, the EC- and EGCG-remodeled $\mathrm{A} \beta_{n}$ still preserve extended $\beta$-sheets in the membrane (Fig. $2 \mathrm{~h}$ and i, blue), although the relative amounts are decreased in the presence of EC and EGCG, in that order (Fig. 2j). In contrast, neither of the catechin-remodelled $A \beta_{n}$ exhibit packing of $\beta$ sheet layers (Fig. 2k, green and blue), in agreement with our ThT data (Fig. 2h). Overall, these findings suggest that the toxic $\mathrm{A} \beta_{n}$ formed under our conditions colocalize, interact and insert into lipid membranes wherein they adopt $\beta$-sheet structures. To identify the toxic $A \beta_{n}$ surfaces that facilitate these multivalent interactions with the membrane, we comparatively examined the ${ }^{15} \mathrm{~N}$-DEST differences between canonical, EC- and EGCGremodeled $A \beta_{n}$ in the presence of model membranes (Fig. 3n, ESI Fig. S2 $\dagger$ ).
Toxic vs. non-toxic $A \beta$ assemblies in the membrane environment exhibit marked differences in $A \beta$-recognition profiles

To focus on the effects of the catechins, the canonical A $\beta_{n}$ DEST profile (ESI Fig. S2b $\dagger$ ) was subtracted from the catechinremodeled $A \beta_{n}$ DEST profiles (ESI Fig. S2c and $d \dagger$ ). Since all profiles in ESI Fig. S2b-d $\dagger$ were recorded in the presence of SUVs, the resulting DEST differences (Fig. 3n) report primarily on the catechin-induced remodeling of $A \beta$ monomer- $A \beta_{n}$ contacts. Specifically, the EGCG-remodeled vs. canonical $\mathrm{A} \beta_{n}$ ${ }^{15} \mathrm{~N}-\Theta$ profile differences $\left(\Delta_{\mathrm{EGCG}} \Theta\right)$ show significant decreases in $\Theta$ in the two $\beta$-strand regions typically observed in $\mathrm{A} \beta$ protofibrils (Fig. 3n, darkblue). These losses are consistent with the $\mathrm{A} \beta$ monomers being less engaged with the $\mathrm{A} \beta_{n}$ surface at the two $\beta$-strand sites in the presence of EGCG. However, the EGCGinduced disengagement detected for the $\beta 1$ and $\beta 2$ regions does not extend to the N-terminal segment, for which a significant enhancement in direct contacts is observed (Fig. 3n, darkblue). A similar N-terminal $\Theta$ DEST enhancement is observed also upon EC addition (Fig. 3n, lightblue), albeit with reduced magnitude (Fig. 3n, light vs. darkblue). Likewise, in the $\beta 1$ region the EC-remodeled $A \beta_{n}$ show $\Theta$ losses with a reduced extent compared to the EGCG-remodeled $\mathrm{A} \beta_{n}$ (Fig. 3n, light $v s$. darkblue). However, the DEST pattern observed for the $\mathrm{N}$ terminal and $\beta 1$ regions does not extend to the $\beta 2$ segment, 
for which EC and EGCG result in opposite $\Theta$ changes (Fig. 3n, light vs. darkblue). These findings imply that exposure of the hydrophobic $\beta 1$-turn region and concomitant shielding of the $\mathrm{N}$-terminus are two key structural transitions intimately linked to toxicity, as these toxic surfaces modulate interactions with the membrane.

\section{Selection of molecular determinants of $A \beta_{n}$ toxicity}

In order to systematically isolate the $A \beta_{n}$ features relevant for toxicity, we identified groups of coupled $A \beta_{n}$ observables by relying on the data correlation matrix (Fig. 4a), whose elements represent the absolute Pearson's correlation coefficients $(|r|)$ between each pair of $\mathrm{A} \beta_{n}$ observables (ESI $\dagger$ ). Through agglomerative clustering of the correlation matrix, we then built a dendrogram that partitions the $\mathrm{A} \beta_{n}$ observables into five distinct clusters (Fig. 4b). The largest cluster, denoted as cluster 1 , includes the $\Delta_{\text {Cat }} \Theta_{i}$ values for residues in the 3-28 region as well as three low resolution observables, i.e. the membraneembedded $\beta$-sheet, the size and the surface hydrophobicity. Since these measurables rank similarly to the relative toxicities (Fig. 1), we hypothesized that cluster 1 defines key molecular determinants of $\mathrm{A} \beta_{n}$ toxicity. This hypothesis is confirmed by two independent lines of evidence.
First, if we re-compute the correlation matrix and agglomerative clustering after including the relative toxicities (Fig. 1a), we find that the toxicity partitions within cluster 1 (ESI Fig. S6 $\dagger$ ), confirming that the observables in this cluster scale with $\mathrm{A} \beta_{n}$ toxicity. Second, in the $\Delta_{\mathrm{EGCG}} \Theta_{i}$ vs. $\Delta_{\mathrm{EC}} \Theta_{i}$ plot (Fig. 4c), the cluster 1 residues fall at or near the region expected to scale with the relative EC vs. EGCG cell viability (CV) data, defined as $\left(\mathrm{CV}_{\mathrm{A} \beta_{40}+\mathrm{EC}}-\mathrm{CV}_{\mathrm{A} \beta_{40}}\right) /\left(\mathrm{CV}_{\mathrm{A} \beta_{40}+\mathrm{EGCG}}-\mathrm{CV}_{\mathrm{A} \beta_{40}}\right)=0.42 \pm 0.05$ (shaded blue area, Fig. 4c). The linear regression of $\Delta_{\mathrm{EGCG}} \Theta_{i} v s$. $\Delta_{\mathrm{EC}} \Theta_{i}$ for cluster 1 is in fact in excellent agreement with the value expected based on the relative cellular viability (dashed blue line with slope of $0.42 \pm 0.02$ and correlation coefficient of 0.98; Fig. 4c). Hence, we conclude that cluster 1 (blue dendrogram in Fig. $4 \mathrm{~b}$ and blue circles in Fig. 4c) is relevant for the toxicity of $\mathrm{A} \beta_{n}$.

To gain further insight on the significance of the $\Delta_{\mathrm{EGCG}} \Theta_{i} v s$. $\Delta_{\mathrm{EC}} \Theta_{i}$ plot and independently corroborate the residue clusters obtained through the agglomerative clustering analysis, we also performed Singular Value Decomposition (SVD) of the data in Fig. 4c. The SVD analysis reveals that the first principal component (dashed black line, Fig. 4c), which accounts for $88 \%$ of the total variance, not only resides within the range expected to scale with the relative cellular viability (i.e. within the shaded
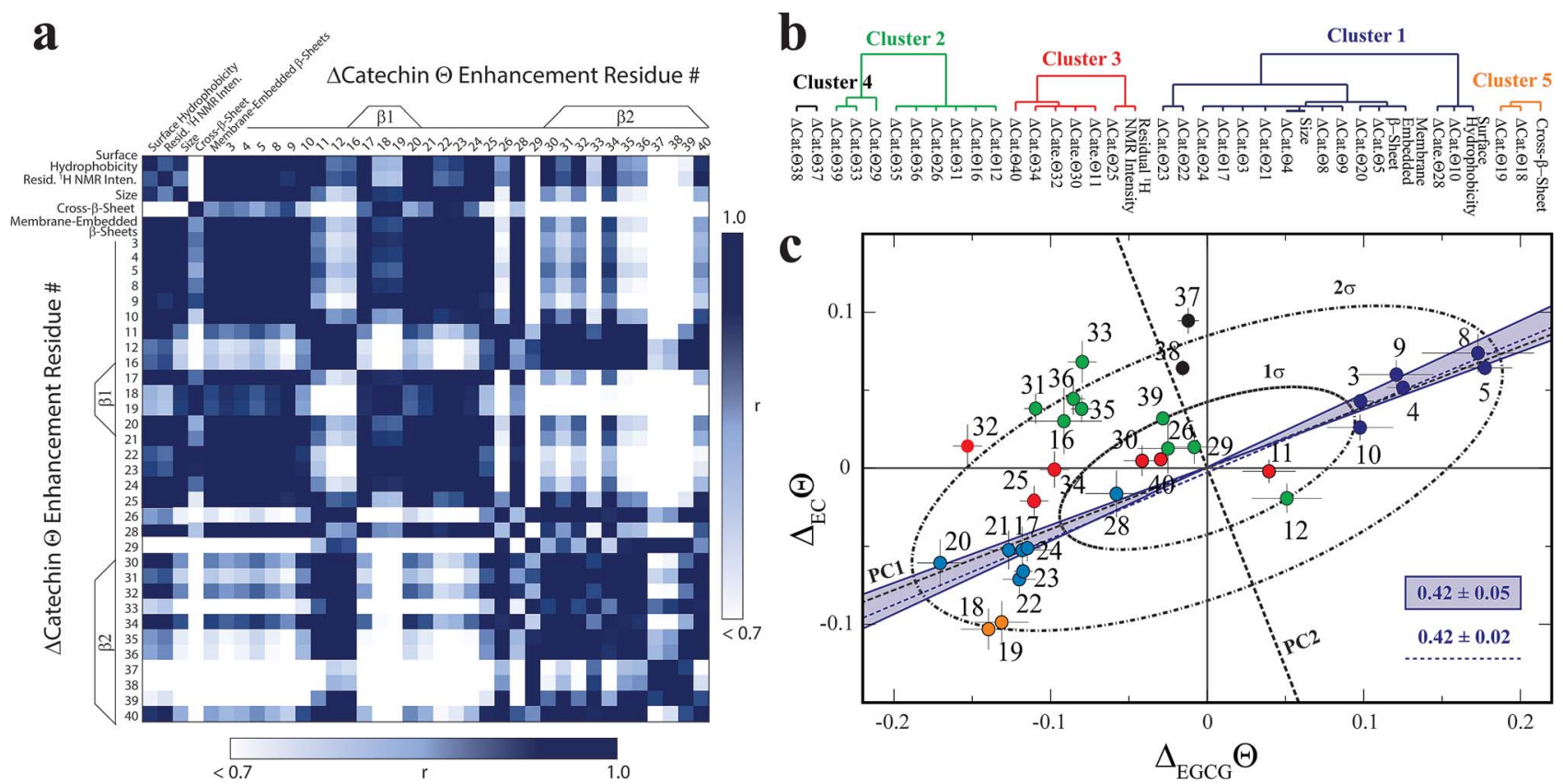

Fig. 4 Identification of the determinants of $A \beta$ assembly toxicity through agglomerative clustering and Singular Value Decomposition (SVD). (a) Correlation matrix for the $A \beta_{n}$ observables from Fig. 1-3. Correlations with an absolute Pearson's correlation coefficient > 0.95 are indicated in dark blue. (b) Dendrogram displaying the clusters with an absolute Pearson's correlation coefficient $>0.9$ obtained through complete linkage agglomerative clustering. (c) Singular Value Decomposition (SVD) of the ${ }^{15} \mathrm{~N}-\mathrm{DEST}$ data. The dashed black lines indicate the first and second principal components (PC1 and PC2) obtained through the SVD of the column-mean centered $\left(\Delta_{\mathrm{EC}} \Theta_{i}, \Delta_{\mathrm{EGCG}} \Theta_{i}\right)$ matrix, where $i$ is the residue number. The ellipsoids at one and two standard deviations for the residue scores along PC1 and PC2 are shown as black dot-dashed curves. Data for residues assigned to clusters 1,2 and 3, 4 and 5 though agglomerative clustering are displayed as solid dark/light blue, green, red, black and orange circles, respectively, and the corresponding residue number is reported beside each circle. The solid blue lines define the region of the $\left(\Delta_{\mathrm{EC}} \Theta_{i}, \Delta_{\mathrm{EGCG}} \Theta_{i}\right)$ plane that is expected to scale with the relative cellular viability $(C V)$ defined as $\left(C V_{\mathrm{A} \beta_{40}+E C}-\mathrm{CV}_{\mathrm{A} \beta_{40}}\right) /\left(C V_{\mathrm{A} \beta_{40}+E G C G}-C V_{A \beta_{40}}\right)=$ $0.42 \pm 0.05$, based on the data of Fig. 1. The dashed blue line (slope of $0.42 \pm 0.02$ and correlation coefficient of 0.98 ) was obtained from the linear regression of the DEST data in cluster 1 (blue) and confirms that cluster 1 correlates with cellular viability. PC1 (slope of 0.39) aligns with the residues for cluster 1. 
$\mathbf{a}$

$$
\begin{gathered}
\mathrm{A} \beta_{\text {assembly }}-\text { Membrane Colocalization \& Insertion } \\
\left(T E M, D L S, W A X D, \Delta^{15} N-R_{2}, S T D H S Q C\right)
\end{gathered}
$$

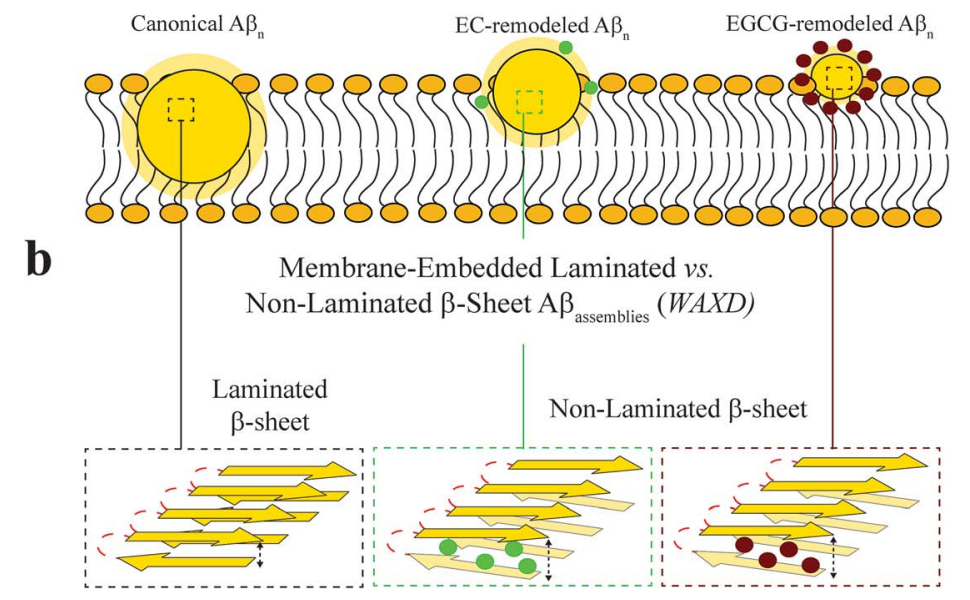

C Monomer Exchange Dynamics on the surface of $\mathrm{A} \beta_{\text {assemblies }}\left({ }^{15} N-D E S T N M R\right)$

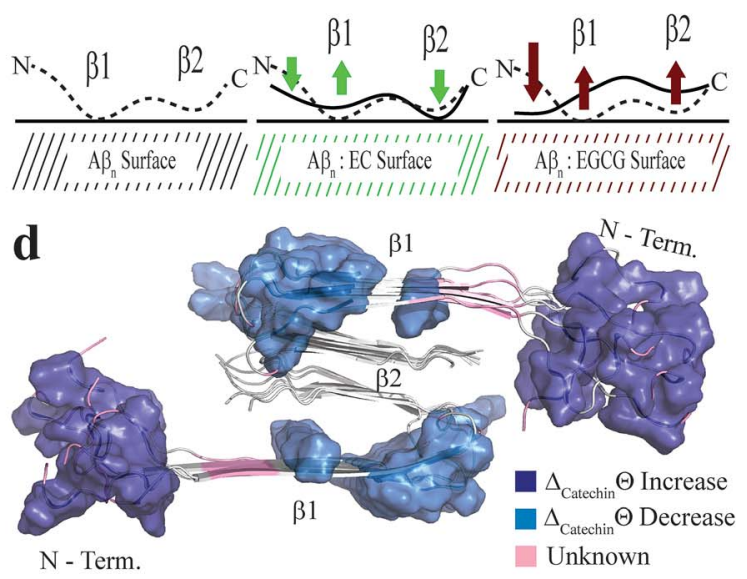

Fig. 5 Proposed model for the molecular determinants of $A \beta$ assembly toxicity. (a) Toxic $A \beta_{n}$ (canonical $A \beta_{n}$ ) exhibit significant solvent exposure of hydrophobic surfaces (yellow glow surrounding $A \beta_{n}$ ). Exposed hydrophobic surfaces facilitate the colocalization, interaction and subsequent insertion of $A \beta_{n}$ into the membrane. (b) Membrane-embedded $A \beta_{n}$ adopt both laminated and non-laminated $\beta$-sheets, indicating that under our experimental conditions the non-laminated $\beta$-sheet signature is the minimum structural feature required for membrane insertion and induction of toxicity. (c) Toxic vs. non-toxic $A \beta_{n}$ exhibit unique regiospecific differences in the recognition of $A \beta$ monomers within a membrane environment. Relative to canonical $A \beta_{n}$ (black), EC- (green) and EGCG-remodeled $A \beta_{n}$ (maroon) exhibit progressive engagement of contacts with $A \beta$ monomers at the $\mathrm{N}$-terminus and disengagement at the $\beta 1$-turn region, following the same ranking as their measured toxicities. In contrast, for the $\beta 2$ region no correlation is observed between toxicity and $A \beta_{n}$ monomer recognition. Relevant experimental techniques are indicated in parenthesis. (d) Mapping on the structure of $A \beta_{40}$ fibrils ${ }^{57}$ (PDB code: $2 L M N$ ) the $A \beta$ residues in cluster 1 (Fig. $4 \mathrm{~b}$ and $c$ ). The $N$-terminal and $\beta 1$-turn residues that correlate with toxicity (blue) are found in the external regions of the A $\beta$ fibril structure. In contrast, $\beta 2$ is involved in the lamination of multiple $\beta$-sheet layers and is largely inaccessible (Table S2†), explaining its ancillary role in toxicity.

blue area in Fig. 4c), but also aligns with the residues for cluster 1. Interestingly, the SVD reveals that cluster 1 (blue circles, Fig. 4c) is composed of two distinct sub-sets that are mostly confined at opposite extremes of PC1, between the $1 \sigma$ and $2 \sigma$ ellipsoids (Fig. 4c). The sub-set with positive PC1 components (dark blue circles) represents the N-terminal residues that become engaged in monomer recognition, as probed by DEST, when cellular viability is enhanced. On the contrary, the cluster 1 sub-set with negative PC1 scores (light blue circles) arises from the $\beta 1$-turn region residues that become engaged when cellular viability decreases.

In stark contrast to cluster 1 , the other clusters obtained from the agglomerative clustering analysis (Fig. 4b, black, green, red and orange circles) fall outside the range expected to scale with cellular viability (blue shaded area, Fig. 4c) and exhibit components along PC2 that are overall higher than those observed for cluster 1 (Fig. 4c). In conclusion, the combined analyses of the correlation matrix, agglomerative clustering and SVD consistently identify the constituents of cluster 1, i.e. surface hydrophobicity, size, membraneembedded $\beta$-sheets, $\mathrm{N}$-terminal residue disengagement and $\beta 1$-turn region engagement, as key molecular determinants of $\mathrm{A} \beta_{n}$ toxicity.

In order to verify the predictive power of the correlation between $A \beta_{n}$ toxicity and cluster 1 , we measured the relative toxicities for the $A \beta$ assemblies not included in Fig. 1a and we compared them to those predicted by our model (Fig. 4; ESI Fig. S7 $\dagger$ ). These $A \beta_{n}$ toxicities were not used to train our model and hence provide a critical test of its prognostic capacity. As seen in ESI Fig. S7d, $\uparrow$ a strong linear correlation is observed between the predicted and observed toxicities $(r \geq 0.94)$, with a slope within error to one, thus validating the predictive power of our model.

In summary, our investigation of the $\mathrm{A} \beta_{n}$ library through the comparative analysis of ${ }^{15} \mathrm{~N}-\mathrm{R}_{2}$ and DEST NMR combined with WAXD, TEM, DLS and extrinsic fluorescence reveals key structural differences that distinguish toxic $v s$. non-toxic $\mathrm{A} \beta$ assemblies. The integrated analyses of our data through agglomerative clustering and SVD consistently identify a cluster of molecular attributes unique to toxic $\mathrm{A} \beta_{n}$ (Fig. $4 \mathrm{~b}$, cluster 1), including surface hydrophobicity, size, membrane-embedded $\beta$-sheets, shielding of the $\mathrm{N}$-terminus and simultaneous exposure of the $\beta 1$-turn region to $A \beta$ monomers, as probed through DEST NMR.

Our data shows that toxic $A \beta_{n}$ exhibit solvent exposed hydrophobic sites accessible to ANS binding. While the relationship between surface hydrophobicity and toxicity has been observed previously for several protein systems such as the Type $\mathrm{A} / \mathrm{B}$ HypF-N assemblies, ${ }^{51,52}$ the $\mathrm{A}^{+} / \mathrm{A}^{-} \mathrm{A} \beta_{42}$ oligomer pair, ${ }^{53}$ the sup35p oligomer pair, ${ }^{54}$ and others, ${ }^{55}$ here we not only systematically confirm this association for the $A \beta$ system using a library of $A \beta$ assemblies, but we also propose an unprecedented mechanism of $A \beta_{n}$ toxicity probed at multiple degrees of resolution. Such mechanism reveals how hydrophobic exposure relates to $A \beta$-membrane interactions and $A \beta$ monomer recognition. The combination of our TEM, DLS and ${ }^{15} \mathrm{~N}$-DEST and $\mathrm{R}_{2}$ 
data collectively shows that $\mathrm{A} \beta_{n}$ with greater surface hydrophobicity e.g. canonical and EC-remodeled $\mathrm{A} \beta_{n}$ colocalize and interact with the membrane surface more effectively than the less toxic $\mathrm{A} \beta_{n}$ with less exposed hydrophobic sites $e . g$. the EGCGremodeled $\mathrm{A} \beta_{n}$ (Fig. 5a).

The surface hydrophobicity-mediated interactions with the membrane are not limited to the membrane surface, as our WAXD data show that canonical and EC-remodeled $\mathrm{A} \beta_{n}$ exhibit significant populations of $\beta$-sheets embedded in the membrane compared to EGCG-remodeled $\mathrm{A} \beta_{n}$. The functional effect of the membrane-embedded $\beta$-sheets is recapitulated by our propidium iodide-based assay, which indicates that canonical $\mathrm{A} \beta_{n}$ significantly enhance the permeability of the cell membrane compared to the less toxic $A \beta_{n}$ formed in the presence of EGCG.

Notably, we also found that cross- $\beta$-sheet structures are dispensable for membrane insertion, as only canonical $\mathrm{A} \beta_{n}$ exhibit cross lamination of $\beta$-sheet layers, whereas ECremodeled $A \beta_{n}$, with comparable levels of membraneembedded $\beta$-sheets, exhibit considerably reduced cross lamination, similar to EGCG remodeled $\mathrm{A} \beta_{n}$ (Fig. $2 \mathrm{k}$ and $5 \mathrm{~b}$ ). The lack of correlation between toxicity and $\beta$-sheet crosslamination is also consistent with the variability in sheet-tosheet pairing angles reported for oligomers of model amyloidogenic sequences stabilized by macrocyclic peptides. ${ }^{56}$

The correlation and SVD analyses also identify a cluster of residues confined to the $\mathrm{N}$-terminus and $\beta 1$-loop region that are key to the regulation of $\mathrm{A} \beta_{n}$ toxicity (Fig. $4 \mathrm{~b}$ and c, cluster 1 ). The probability distribution of contacts between $A \beta$ monomers and the $\mathrm{A} \beta_{n} /$ SUVs surface is markedly enhanced in the $\beta 1$-loop region (residues 17-28) and concomitantly reduced at the $\mathrm{N}$ terminal segment (residues $3-10$ ) as the $\mathrm{A} \beta_{n}$ toxicity increases (Fig. $3 \mathrm{n}$ and $5 \mathrm{c}$, green vs. maroon arrows). Interestingly, an unexpected decorrelation with toxicity is observed at the $\beta 2$ region (residues 30-40) (clusters 2 and 4), for which the ECremodeled $\mathrm{A} \beta_{n}$, with intermediate toxicity, exhibits a further enhancement in contacts relative to the canonical $\mathrm{A} \beta_{n}$ (Fig. $3 \mathrm{n}$ and $5 \mathrm{c}$, green), in stark contrast to the reduction observed for EGCG-remodeled $\mathrm{A} \beta_{n}$ (Fig. $3 \mathrm{n}$ and $5 \mathrm{c}$, green vs. maroon arrows).

Notably, the $\mathrm{N}$-terminus and $\beta 1$-loop $\mathrm{A} \beta$ regions identified by the correlation and SVD analyses to be toxicity determinants (Fig. 4c, cluster 1) are located at the external surface of the $A \beta_{40}$ fibril structure (Fig. 5d, blue surfaces). Furthermore, most familial AD mutations (English, Tottori, Iowa, Arctic, Dutch and Italian) that alter the biophysical properties of $A \beta$ are observed in the N-terminal and $\beta 1$ regions. ${ }^{\mathbf{1 , 5 8}}$ Conversely, the $\beta 2$ region not identified by SVD as linked to toxicity, is inaccessible to the environment (Table $\mathrm{S} 2 \dagger$ ) and is found embedded into the structural core of the fibril, where it is involved in the cross lamination of multiple $\beta$-sheet layers (Fig. $5 \mathrm{~d}$, grey cartoon). These observations agree with our WAXD and ThT data, consistently pointing to $\beta$-sheet lamination as accessory to toxicity induction.

\section{Conclusions}

Overall, our data indicate that $\mathrm{A} \beta_{n}$ toxicity is regulated by the solvent exposure of hydrophobic surfaces, wherein the hydrophobic $\beta 1$-turn region is more accessible to monomer/ SUV recognition, while the highly charged N-terminus is shielded from such recognition. In comparison, the role of $\beta 2$ appears to be largely ancillary. These toxic surfaces enhance the colocalization, contacts and subsequent insertion of $\beta$-sheet rich $\mathrm{A} \beta_{n}$ into the membrane, leading to compromised membrane stability. Moreover, the proposed model is able to predict relative toxicities solely based on low-resolution measurements, such as size and surface hydrophobicity. Modulation of these properties through small-molecule treatment can be utilized as an effective strategy to reduce the toxicity associated with soluble $\mathrm{A} \beta$ assemblies. In addition, soluble oligomers of amyloidogenic peptides with different sequences have been suggested to share a common conformation, ${ }^{59}$ and $A \beta$ is not only relevant for dominantly inherited $A D$, but also serves as a model system for a broad-range of amyloid disorders. Hence, the cluster of molecular attributes identified here to correlate with toxicity may be transferrable to other amyloidogenic systems.

\section{Author contributions}

R. A. and G. M. designed research; R. A., M. A., A. K., J. B. and V. H. performed research; R. A., A. K., M. R., J. B., R. E., V. H., R. W., S. B., J. H., C. V. and G. M. analyzed data; R. A. and G. M. wrote the paper. All authors have given approval to the final version of the manuscript. This project was funded by the Natural Sciences and Engineering Research Council of Canada (NSERC RGPIN-2019-05990; RGPIN-2018-05585; RGPIN-2016-06450; RGPIN-2014-04514).

\section{Conflicts of interest}

There are no conflicts to declare.

\section{Acknowledgements}

We thank M. Akimoto, B. VanSchouwen, N. Jafari, Y. Lu, R. Truant (McMaster U.), M. H. Lim (KAIST) and S. J. Cox (U. Michigan) for helpful discussions.

\section{Notes and references}

1 I. Benilova, E. Karran and B. de Strooper, Nat. Neurosci., 2012, 15, 349-357.

2 J. A. Hardy and G. A. Higgins, Science, 1992, 256, 184-185.

3 E. S. Musiek and D. M. Holtzman, Nat. Neurosci., 2015, 18, 800-806.

4 C. R. Jack, D. S. Knopman, W. J. Jagust, R. C. Petersen, M. W. Weiner, P. S. Aisen, L. M. Shaw, P. Vemuri, H. J. Wiste, S. D. Weigand, T. G. Lesnick, V. S. Pankratz, M. C. Donohue and J. Q. Trojanowski, Lancet Neurol., 2013, 12, 207-216.

5 J. M. Castellano, J. Kim, F. R. Stewart, H. Jiang, R. B. DeMattos, B. W. Patterson, A. M. Fagan, J. C. Morris, K. G. Mawuenyega, C. Cruchaga, A. M. Goate, K. R. Bales, 
S. M. Paul, R. J. Bateman and D. M. Holtzman, Sci. Transl. Med., 2011, 3, 89ra57.

6 R. D. Terry, E. Masliah, D. P. Salmon, N. Butters, R. DeTeresa, R. Hill, L. A. Hansen and R. Katzman, Ann. Neurol., 1991, 30, 572-580.

7 W. L. Klein, G. A. Krafft and C. E. Finch, Trends Neurosci., 2001, 24, 219-224.

8 J. L. Tomic, A. Pensalfini, E. Head and C. G. Glabe, Neurobiol. Dis., 2009, 35, 352-358.

9 M. Jin, N. Shepardson, T. Yang, G. Chen, D. Walsh and D. J. Selkoe, Proc. Natl. Acad. Sci. U. S. A., 2011, 108, 58195824.

10 Y. Miller, B. Ma and R. Nussinov, Biophys. J., 2009, 97, 11681177.

11 K. Ono, M. M. Condron and D. B. Teplow, Proc. Natl. Acad. Sci. U. S. A., 2009, 106, 14745-14750.

12 P.-N. Cheng, C. Liu, M. Zhao, D. Eisenberg and J. S. Nowick, Nat. Chem., 2012, 4, 927-933.

13 R. Riek and D. S. Eisenberg, Nature, 2016, 539, 227-235.

14 M. Kinoshita, E. Kakimoto, M. S. Terakawa, Y. Lin, T. Ikenoue, M. So, T. Sugiki, A. Ramamoorthy, Y. Goto and Y.-H. Lee, Phys. Chem. Chem. Phys., 2017, 19, 16257-16266.

15 J. D. Gehman, R. Verardi, A. K. Mehta, G. Veglia and F. Separovic, Biophys. J., 2010, 98, 176a.

16 Y. Miller, B. Ma, C.-J. Tsai and R. Nussinov, Proc. Natl. Acad. Sci. U. S. A., 2010, 107, 14128-14133.

17 H. A. Lashuel, D. Hartley, B. M. Petre, T. Walz and P. T. Lansbury, Nature, 2002, 418, 291.

18 A. Quist, I. Doudevski, H. Lin, R. Azimova, D. Ng, B. Frangione, B. Kagan, J. Ghiso and R. Lal, Proc. Natl. Acad. Sci. U. S. A., 2005, 102, 10427-10432.

19 M. F. M. Sciacca, S. A. Kotler, J. R. Brender, J. Chen, D.-k. Lee and A. Ramamoorthy, Biophys. J., 2012, 103, 702-710.

20 M. Ahmed, J. Davis, D. Aucoin, T. Sato, S. Ahuja, S. Aimoto, J. I. Elliott, W. E. van Nostrand and S. O. Smith, Nat. Struct. Mol. Biol., 2010, 17, 561-567.

21 S. Chimon, M. A. Shaibat, C. R. Jones, D. C. Calero, B. Aizezi and Y. Ishii, Nat. Struct. Mol. Biol., 2007, 14, 1157-1164.

22 S. J. C. Lee, E. Nam, H. J. Lee, M. G. Savelieff and M. H. Lim, Chem. Soc. Rev., 2017, 46, 310-323.

23 C. A. Ross and M. A. Poirier, Nat. Rev. Mol. Cell Biol., 2005, 6, 891-898.

24 S. A. Kotler, P. Walsh, J. R. Brender and A. Ramamoorthy, Chem. Soc. Rev., 2014, 43, 6692-6700.

25 F. Chiti and C. M. Dobson, Annu. Rev. Biochem., 2017, 86, 2768.

26 T. Kawarabayashi, M. Shoji, L. H. Younkin, L. Wen-Lang, D. W. Dickson, T. Murakami, E. Matsubara, K. Abe, K. H. Ashe and S. G. Younkin, J. Neurosci., 2004, 24, 38013809.

27 E. E. Ambroggio, D. H. Kim, F. Separovic, C. J. Barrow, K. J. Barnham, L. A. Bagatolli and G. D. Fidelio, Biophys. J., 2005, 88, 2706-2713.

28 J. W. Um, H. B. Nygaard, J. K. Heiss, M. A. Kostylev, M. Stagi, A. Vortmeyer, T. Wisniewski, E. C. Gunther and S. M. Strittmatter, Nat. Neurosci., 2012, 15, 1227-1235.
29 N. L. Fawzi, J. Ying, R. Ghirlando, D. A. Torchia and G. M. Clore, Nature, 2011, 480, 268-272.

30 J. Bieschke, J. Russ, R. P. Friedrich, D. E. Ehrnhoefer, H. Wobst, K. Neugebauer and E. E. Wanker, Proc. Natl. Acad. Sci. U. S. A., 2010, 107, 7710-7715.

31 E. Sironi, L. Colombo, A. Lompo, M. Messa, M. Bonanomi, M. E. Regonesi, M. Salmona and C. Airoldi, Chemistry, 2014, 20, 13793-13800.

32 C. Visentin, F. Pellistri, A. Natalello, J. Vertemara, M. Bonanomi, E. Gatta, A. Penco, A. Relini, L. de Gioia, C. Airoldi, M. E. Regonesi and P. Tortora, Hum. Mol. Genet., 2017, 26, 3271-3284.

33 M. Xu, D. J. McCanna and J. G. Sivak, J. Pharmacol. Toxicol. Methods, 2015, 71, 1-7.

34 H. Lecoeur, Exp. Cell Res., 2002, 277, 1-14.

35 S. A. Latt and G. Stetten, J. Histochem. Cytochem., 1976, 24, 24-33.

36 B. Mannini, E. Mulvihill, C. Sgromo, R. Cascella, R. Khodarahmi, M. Ramazzotti, C. M. Dobson, C. Cecchi and F. Chiti, ACS Chem. Biol., 2014, 9, 2309-2317.

37 F. L. Palhano, J. Lee, N. P. Grimster and J. W. Kelly, J. Am. Chem. Soc., 2013, 135, 7503-7510.

38 J. M. Lopez del Amo, U. Fink, M. Dasari, G. Grelle, E. E. Wanker, J. Bieschke and B. Reif, J. Mol. Biol., 2012, 421, 517-524.

39 T. L. Williams and L. C. Serpell, FEBS J., 2011, 278, 39053917.

40 R. Ahmed and G. Melacini, Chem. Commun., 2018, 54, 46444652.

41 J. Milojevic, V. Esposito, R. Das and G. Melacini, J. Am. Chem. Soc., 2007, 129, 4282-4290.

42 G. Fusco, S. W. Chen, P. T. F. Williamson, R. Cascella, M. Perni, J. A. Jarvis, C. Cecchi, M. Vendruscolo, F. Chiti, N. Cremades, L. Ying, C. M. Dobson and A. de Simone, Science, 2017, 358, 1440-1443.

43 G. Fusco, T. Pape, A. D. Stephens, P. Mahou, A. R. Costa, C. F. Kaminski, G. S. Kaminski Schierle, M. Vendruscolo, G. Veglia, C. M. Dobson and A. de Simone, Nat. Commun., 2016, 7, 12563.

44 G. Fusco, A. de Simone, T. Gopinath, V. Vostrikov, M. Vendruscolo, C. M. Dobson and G. Veglia, Nat. Commun., 2014, 5, 3827.

45 N. L. Fawzi, J. Ying, D. A. Torchia and G. M. Clore, J. Am. Chem. Soc., 2010, 132, 9948-9951.

46 C. R. Bodner, C. M. Dobson and A. Bax, J. Mol. Biol., 2009, 390, 775-790.

47 C. R. Bodner, A. S. Maltsev, C. M. Dobson and A. Bax, Biochemistry, 2010, 49, 862-871.

48 A. Ceccon, V. Tugarinov, A. Bax and G. M. Clore, J. Am. Chem. Soc., 2016, 138, 5789-5792.

49 M. Algamal, R. Ahmed, N. Jafari, B. Ahsan, J. Ortega and G. Melacini, J. Biol. Chem., 2017, 292, 17158-17168.

50 R. Ahmed, B. VanSchouwen, N. Jafari, X. Ni, J. Ortega and G. Melacini, J. Am. Chem. Soc., 2017, 139, 13720-13734.

51 S. Campioni, B. Mannini, M. Zampagni, A. Pensalfini, C. Parrini, E. Evangelisti, A. Relini, M. Stefani, 
C. M. Dobson, C. Cecchi and F. Chiti, Nat. Chem. Biol., 2010, 6, 140-147.

52 C. Capitini, J. R. Patel, A. Natalello, C. D'Andrea, A. Relini, J. A. Jarvis, L. Birolo, A. Peduzzo, M. Vendruscolo, P. Matteini, C. M. Dobson, A. de Simone and F. Chiti, Chem. Commun., 2018, 54, 8637-8640.

53 A. R. A. Ladiwala, J. Litt, R. S. Kane, D. S. Aucoin, S. O. Smith, S. Ranjan, J. Davis, W. E. van Nostrand and P. M. Tessier, J. Biol. Chem., 2012, 287, 24765-24773.

54 R. Krishnan, J. L. Goodman, S. Mukhopadhyay, C. D. Pacheco, E. A. Lemke, A. A. Deniz and S. Lindquist, Proc. Natl. Acad. Sci. U. S. A., 2012, 109, 11172-11177.

55 B. Bolognesi, J. R. Kumita, T. P. Barros, E. K. Esbjorner, L. M. Luheshi, D. C. Crowther, M. R. Wilson,
C. M. Dobson, G. Favrin and J. J. Yerbury, ACS Chem. Biol., 2010, 5, 735-740.

56 J. Zheng, C. Liu, M. R. Sawaya, B. Vadla, S. Khan, R. J. Woods, D. Eisenberg, W. J. Goux and J. S. Nowick, J. Am. Chem. Soc., 2011, 133, 3144-3157.

57 A. T. Petkova, Y. Ishii, J. J. Balbach, O. N. Antzutkin, R. D. Leapman, F. Delaglio and R. Tycko, Proc. Natl. Acad. Sci. U. S. A., 2002, 99, 16742-16747.

58 C. de Jonghe, Hum. Mol. Genet., 2001, 10, 1665-1671.

59 R. Kayed, E. Head, J. L. Thompson, T. M. McIntire, S. C. Milton, C. W. Cotman and C. G. Glabe, Science, 2003, 300, 486-489. 